\title{
CARACTERIZAÇÃO FITOSSOCIOLÓGICA EM ÁREAS DE OCORRÊNCIA NATURAL DE CANDEIA (Eremanthus erythropappus (D.C.) MacLeish) ${ }^{1}$
}

Agostinho Lopes de Souza ${ }^{2}$, Marcio Leles Romarco de Oliveira $^{3}$, Elisiane Fátima da Silva ${ }^{4}$, Danilo José da Silva Coelho ${ }^{3}$

\begin{abstract}
RESUMO - A candeia vem sendo submetida a uma exploração desordenada, devido ao seu elevado potencial econômico. A solução para o controle da exploração de forma desordenada é o desenvolvimento e a execução de planos de manejo florestal, sendo, o conhecimento da estrutura de Eremanthus erythropappus (candeia), tanto na forma específica quanto na forma florestal, de fundamental importância para o desenvolvimento de um manejo adequado. Os objetivos deste trabalho foram estudar a estrutura de E. erythropappus, em diferentes ambientes, e caracterizar os parâmetros das estruturas horizontal, vertical e interna e as distribuições de diâmetro, área basal e volume da espécie. Foram utilizados dados de cinco áreas: as áreas 1, 2 e 3, maciços de candeia na forma específica; área 4, ocorrência rara de candeia; e área 5, ocorrência de candeia na forma florestal. Foram amostradas cinco parcelas de $400 \mathrm{~m}^{2}$ distribuídas sistematicamente em cada área. Após as análises, verificou-se que com o decorrer do tempo, cresce a população de outras espécies e decresce a população de candeia. A área 1 apresentou maiores valores de densidade absoluta, enquanto os melhores indivíduos de candeia, tanto em tamanho quanto em qualidade de fuste, ocorreram na área 5. Os indivíduos arbóreos das áreas estudas foram agrupados em dois estratos de altura total, porém os limites dos estratos verticais diferiram de uma área para outra. A distribuição da densidade $\left(n h a^{-1}\right)$ por classe de diâmetro para a comunidade arbórea e considerando apenas os indivíduos de candeia não diferiu significativamente pelo teste de Qui-quadrado.
\end{abstract}

Palavras-chave: Estrutura fitossociológica, Itacolomi e distribuição diamétrica

\section{PHYTOSSOCIOLOGICAL CHARACTERIZATION IN AREAS OF NATURAL OCCURRENCE OF CANDEIA (Eremanthus erythropappus (D.C.) MacLeish)}

\begin{abstract}
Candeia (Eremanthus erythropappus) has been intensely exploited, because of its high economic importance. The solution for controlling this disordered exploitation is the development and implementation offorest management projects, and knowledge on candeia structure, both in the specific form and forest form, is crucial for the development of correct management. The objective of this work was to study the structure of Eremanthus erythropappus in different environments and characterize the parameters of the horizontal, vertical and internal structures and the distribution of diameter, basal area and volume of the species. Data were obtained from 5 sites; sites 1,2 and 3 consisted of candeia populations in the specific form, site 4 with rare occurrences of the species, and site 5 with occurrence of candeia in the forest form. A total of 5 plots, $400 \mathrm{~m}^{2}$, systematically distributed in each site were sampled. The analysis showed that, over the time, the number of other species increases and the number of candeia trees decreases. Site 1 showed the highest absolute density, while the best individuals, both in size and trunk quality, were found in site 5. The tree individuals in the studied sites were grouped in two total height strata, but the vertical strata limits differed among sites.

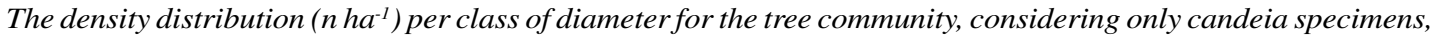
was not significantly different by the $X^{2}$ test.
\end{abstract}

Keywords: Phytossociological structure, Itacolomi and diametric distribution.

\footnotetext{
${ }^{1}$ Recebido em 04.09.2006 e aceito para publicação em 29.03.2007

${ }^{2}$ Departamento de Engenharia Floresta da Universidade Federal de Viçosa (UFV). E-mail: <alsouza@ ufv.br>.

${ }^{3}$ Programa de Pós-Graduação em Ciência Florestal da UFV. E-mail: <marcioromarco@ yahoo.com.br > e <djcoelho@ufv.br>.

${ }^{4}$ Engenheira Florestal, M.Sc. em Ciência Florestal da UFV. E-mail: <effsilva@ bol.com.br>.
} 


\section{INTRODUÇÃO}

As florestas nativas remanescentes do território brasileiro savaguardam grande riqueza e diversidade de espécies vegetais, e dentre essa riqueza sobressai Eremanthus erythropappus (D.C.) MacLeish, que, apesar do seu elevado potencial econômico, é de silvicultura pouco conhecida.

A madeira de E. erythropappus tem alta resistência, durabilidade e poder energético, sobretudo contém um óleo essencial, $\alpha$-bisabolol, cujo princípio ativo tem propriedades antiflogística, antibacteriana e antimicótica e é comumente empregado na indústria de produtos dermatológicos e cosméticos (PEDRALLI, 1997). Contudo, a principal utilização da madeira de E. erythropappus é na produção de estacas e moirões para cercas.

A espécie adapta-se a um clima com duas estações bem definidas: uma seca e fria, compreendendo os meses de maio, junho, julho, agosto e setembro; e outra chuvosa e quente, durante os meses de outubro, novembro, dezembro, janeiro, fevereiro, março e abril (ARAUJO, 1994).

Um candeial corresponde à formação pioneira de E. erythropappus, que se estabelece após a perturbação da floresta. Com o decorrer da sucessão, o número de indivíduos diminui à medida que a floresta se torna mais estruturada (CETEC, 1996). As árvores podem apresentar duas formas distintas: específica - árvores de pequeno porte, com até $10 \mathrm{~m}$ de altura total, fuste irregular e curto; e florestal - árvores de porte médio, $12 \mathrm{~m}$ de altura total, fuste reto, cilíndrico e mais longo em relação à copa. A forma específica resulta da dispersão de sementes muito leves, que são disseminadas pelo vento a distâncias variáveis. A forma florestal é observada nos indivíduos de maior porte somente nos estádios intermediários de sucessão da floresta mesófila, desaparecendo quando o dossel se forma e os indivíduos de outras espécies dominam os da candeia, sombreandoa (PEDRALLI, 1997).

A candeia vem sendo submetida a uma exploração desordenada, devido ao seu potencial econômico. A solução para o controle da exploração é o desenvolvimento e a execução de planos de manejo florestal sustentável fundamentado no conhecimento da ecologia da espécie, de modo que esta possa ser explorada e, ao mesmo tempo, manejada para sustentabilidade. Conhecer a estrutura de $E$. erythropappus, tanto na forma específica quanto na forma florestal, é de fundamental importância para o desenvolvimento de um manejo adequado.

Diante do exposto, o objetivo deste trabalho foi estudar a estrutura de E. erythropappus em diferentes ambientes, caracterizando os parâmetros das estruturas horizontal, vertical e interna e as distribuições de diâmetro, área basal e volume da espécie.

\section{MATERIAL E MÉTODOS}

\subsection{Caracterização da Área}

O estudo foi executado no Parque Estadual do Itacolomi, com área de aproximadamente 7.000 ha, localizado nos Municípios de Mariana e Ouro Preto (20²3'45 de latitude sul e 433' 33 de longitude oeste), distando $110 \mathrm{~km}$ de Belo Horizonte, Estado de Minas Gerais (FEAM, 1995). Conforme Pedralli (1997), a área pertence aos domínios da Floresta Atlântica e dos Cerrados.

O clima da região é temperado úmido, com inverno seco e verão quente e chuvoso e temperaturas médias anuais variando entre 14 e $19^{\circ} \mathrm{C}$ (ANTUNES, 1986). A cobertura vegetal, em sua maior parte, é constituída por floresta estacional semidecidual montana. Os solos são desenvolvidos sobre material litológico, principalmente xistos, filitos e quartzitos. Apresentam caráter raso (solos Litólicos) ou pouco a medianamente profundo (Cambissolos), com presença de minerais primários facilmente intemperizados (CETEC, 1996). De acordo com a classificação de solos da Embrapa (1999), os solos Litólicos passaram a se chamar de Neossolos, e os Cambissolos permaneceram com essa denominação. O relevo é fortemente ondulado e montanhoso, com altitude variando de 700 a $1.772 \mathrm{~m}$ acima do nível do mar (IEF, 2004).

\subsection{Amostragem e Coleta de Dados}

Empregou-se a amostragem estratificada com seleção sistemática das unidades de amostra, sendo definidos cinco estratos: maciços de candeia (Eremanthus erythropappus (D.C.) MacLeish) na forma específica (Áreas 1, 2 e 3), com distintas condições de solos e exposição solar; área onde a ocorrência de candeia é rara (Área 4); e área de mata com ocorrência de candeia (Área 5), que corresponde à sua forma florestal. Foram inventariadas cinco parcelas de área fixa de 20 x $20 \mathrm{~m}\left(400 \mathrm{~m}^{2}\right)$ por estrato, perfazendo 25 parcelas ou 1,0 ha de área amostrada. 
Avaliaram-se todas as árvores com DAP (diâmetro à altura de 1,30 $\mathrm{m}$ do solo) igual ou superior a $3,0 \mathrm{~cm}$. Em cada parcela, para cada indivíduo amostrado foram feitas medições de CAP (circunferência à altura de 1,30 m do solo), com o emprego de fita métrica; estimação das alturas comercial (Hc) e total (Ht), com o auxílio de uma vara de $3,0 \mathrm{~m}$, e também foi avaliada a qualidade do fuste comercial (QF).

\subsection{Estrutura Vertical}

Foi empregada uma técnica de análise multivariada para estratificação das alturas das comunidades, onde as árvores individuais foram organizadas em ordem crescente de altura total, classificadas em classes com amplitude de $1 \mathrm{~m}$, e em seguida foi elaborada uma matriz $\mathbf{X}$ de dados de alturas totais, em que cada variável $\mathbf{x}_{\mathrm{ij}}$ representou a altura total da $\mathbf{i}$-ésima árvore classificada na $\mathbf{j}$-ésima classe de altura. A matriz $\mathbf{X}$ foi o input das análises de agrupamento e discriminante. Utilizaramse a distância euclidiana e o método de ligação completa (SOUZA et al., 2003). Foram estimadas por estrato a DA e, por último, as posições sociológicas absoluta (PSA) e relativa (PSR), mediante o emprego das fórmulas:

$D A_{i}=\frac{n_{i}}{A} \quad P S A_{i}=\sum_{i=1}^{J} \frac{D A_{i}}{D T A} \cdot D A_{i j} \quad P S R_{i}=\sum_{i=1}^{J} \frac{P S A_{i}}{\sum_{i=1}^{J} D A_{i}} \cdot 100$

em que $D A_{i}=$ densidade absoluta da i-ésima espécie, em número de indivíduos por hectare; $D A_{i j}=$ densidade absoluta da i-ésima espécie no j-ésimo estrato de altura, em número de indivíduos por hectare; $n_{i}=$ número de indivíduos da i-ésima espécie na amostragem; $A=$ área total da amostragem, em hectare; $D T A=$ densidade total, em número de indivíduos por hectare; $P S A_{i}=$ posição sociológica absoluta da i-ésima espécie; e $P S R_{i}=$ posição sociológicas relativa da i-ésima espécie PSR.

\subsection{Estimação dos Parâmetros DA, DoA e VoA}

Os dados da vegetação foram primeiramente resumidos em E. erythropappus e outras espécies e, posteriormente, estimados os parâmetros densidade absoluta (DA), densidade relativa (DR), dominância absoluta (DoA), dominância relativa (DoR), volume absoluto (VoA), volume relativo (VoR) e valor de cobertura (VC) (LAMPRECHT, 1964). Para estimar o volume por árvore individual de candeia, foi utilizada a seguinte equação volumétrica (PÉREZ et al., 2004): $\ln \left(v_{i}\right)=-12,021443+2.024449 \times \ln \left(C A P_{i}\right)+0,822959 \times \ln \left(H t_{i}\right), \quad$ com $\overline{R^{2}}=97,63 \% ; S_{y x}= \pm 0,052778 \ln \left(v_{i}\right) ;$ e $S_{\mathrm{yx}}=28,21 \%$

em que: $v_{i}=$ volume total da $\mathbf{i}$-ésima árvore individual, com casca, em $\mathbf{m}^{3} ; C A P_{i}=$ circunferência à altura de $1,30 \mathrm{~m}$ do solo da i-ésima árvore individual, em cm; e $H t=$ altura total da i-ésima árvore individual, em m.

Para estimar o volume por árvore individual de outras espécies, foi utilizada a seguinte equação volumétrica (CETEC, 1995):

$v t_{i}=0,00007523 \times \mathrm{DAP}_{i}^{1,707348} \times \mathrm{Ht}_{i}^{1,16873}, \mathrm{com}$ $\overline{R^{2}}=97,3 \%$

A estimativa do volume absoluto (VoA), em $m^{3} h a^{-1}$, foi obtida pelo emprego da expressão:

$$
V o A=A \times \sum_{i=1}^{N} v_{i}
$$

em que:

$\mathbf{A}$ = área amostrada, em ha; e $\mathbf{N}=$ número total de árvores amostradas na área $\mathrm{A}$.

Para estimar o número de moirões de candeia, foi empregada a seguinte equação:

$$
n m_{i}=\operatorname{Int}\left(\frac{H c_{i}}{2,20}\right)
$$

em que:

$n m_{i}=$ número de moirões da i-ésima árvore individual; $H c_{i}=$ altura comercial da i-ésima árvore individual, em m; $2,20=$ comprimento do moirão, conforme estabelecido em ABNT (1986); e Int = inteiro do resultado da expressão entre parênteses.

A estimativa do número absoluto de moirões (NoM) de candeia, por hectare, foi obtida pelo emprego da seguinte expressão:

$$
N o M=A \times \sum_{i=1}^{N} n m_{i}
$$

Quanto à estrutura interna, avaliou-se a qualidade de fuste comercial, que é um parâmetro que indica o valor econômico do estoque de madeira da espécie, sendo avaliada mediante o emprego da classificação de tronco: $\mathrm{QF}_{1}=$ fuste bom $(80-100 \%) ; \mathrm{QF}_{2}=$ regular $(50-79 \%) ; \mathrm{e}_{3}=$ inferior $(<50 \%)$ (AMARAL et al., 1998).

R. Árvore, Viçosa-MG, v.31, n.4, p.667-677, 2007 
Para avaliar a estrutura diamétrica, as árvores com DAP igual ou superior a $3,0 \mathrm{~cm}$ foram agrupadas em classes de DAP, segundo as normas estabelecidas pela ABNT (1986), para moirões de madeira preservada para cerca. Foram obtidas as estimativas de: número de árvores (DA); área basal (DoA); e volume (VoA), por hectare e por classe de DAP, de E. erythropappus.

\subsection{Procedimentos Estatísticos}

As estimativas médias de número de árvores por hectare de todas as espécies e de E. erythropappus, por área e por classe de DAP, foram comparados entre si, mediante o emprego do teste Qui-quadrado.

\section{RESULTADOS E DISCUSSÃO}

\subsection{Estrutura Horizontal}

A densidade absoluta estimada em número de indivíduos por hectare $\left(n h a^{-1}\right)$ e por área (estrato) foi: Área 1, 2.540 (1.525 $n h a^{-1}$ de E. erythropappus, 725 $n h a^{-1}$ de outras espécies e $290 n h a^{-1}$ mortas); Área 2, 2.385 (830 $n h a^{-1}$ de E. erythropappus, $1.095 n h a^{-1}$ de outras espécies e $460 n h a^{-1}$ mortas); Área 3, 2.420 (755 $n h a^{-1}$ de E. erythropappus, $940 n h a^{-1}$ de outras espécies e $725 n h a^{-1}$ de mortas); Área 4, 2525 (160 $n h a^{-1}$ de E. erythropappus, $2.265 n h a^{-1}$ de outras espécies e $100 n h a^{-1}$ mortas); e Área 5, 5.085 (620 $n h a^{-1} \mathrm{de}$
E. erythropappus, $4.010 n h a^{-1}$ de outras espécies e $455 n h a^{-1}$ mortas). Observou-se que nos povoamentos naturais de candeia na sua forma específica (Áreas $\mathbf{1}, 2$ e 3), enquanto cresce o número de indivíduos mortos e diminui a população de candeia. Nas Áreas 4 e 5 há predominância de outras espécies. Esse fato indica que, em geral, a candeia co-habita nos povoamentos naturais com outras espécies. Contudo, com o decorrer do tempo cresce a população de outras espécies e decresce a população de candeia (Figura 1). Sobretudo os indivíduos de candeia remanescentes nas comunidades de mata natural (Área 5) assumem a forma florestal e são de maior diâmetro e altura total e, provavelmente, boas árvores-matriz (Quadro 1). Esses resultados são coincidentes com os apresentados por Pérez et al. (2004), que constataram também que a espécie E. erythropappus ocorre na mata com outras espécies, mas predomina em maciços homogêneos, sendo importante critério a ser considerado na aplicação de planos de manejo.

Na Área 4 (ocorrência rara de candeia) foram encontrados poucos indivíduos de E. erythropappus, devido à existência, no local, de gramíneas cobrindo o solo, o que dificulta o contato da semente com o solo.

Quadro 1 - Valores de densidade absoluta (DA) e relativa (DR), dominância absoluta (DoA) e relativa (DoR); e valor de cobertura relativo (VC\%), de E. erythropappus, em relação às outras espécies amostradas nas áreas 1, 2, 3, 4 e 5, em setembro de 2000, no Parque Estadual do Itacolomi, em Ouro Preto, MG

Table 1 - Values of absolute density $(D A)$ and relative density $(D R)$, absolute dominance $(D o A)$ and relative dominance $(D o R)$, and relative cover value (VC\%), for E. erythropappus in relation to the other species sampled in the sites $1,2,3,4$ and 5

\begin{tabular}{llccccc}
\hline Área & Espécie & DA & DoA & DR & DoR & VC\% \\
\hline \multirow{3}{*}{01} & E. erythropappus & 1525 & 18,130 & 60,0 & 86,8 & 73,4 \\
& Outras & 1015 & 2,761 & 40,0 & 13,2 & 26,6 \\
& Total & $\mathbf{2 5 4 0}$ & $\mathbf{2 0 , 8 9 1}$ & $\mathbf{1 0 0 , 0}$ & $\mathbf{1 0 0 , 0}$ & $\mathbf{1 0 0 , 0}$ \\
\hline \multirow{3}{*}{02} & E.erythropappus & 830 & 11,745 & 34,8 & 62,2 & 48,5 \\
& Outras & 1555 & 7,153 & 65,2 & 37,8 & 51,5 \\
& Total & $\mathbf{2 3 8 5}$ & $\mathbf{1 8 , 8 9 8}$ & $\mathbf{1 0 0 , 0}$ & $\mathbf{1 0 0 , 0}$ & $\mathbf{1 0 0 , 0}$ \\
\hline \multirow{3}{*}{03} & E. erythropappus & 755 & 16,824 & 31,2 & 65,2 & 48,2 \\
& Outras & 1665 & 8,995 & 68,8 & 34,8 & 51,8 \\
& Total & $\mathbf{2 4 2 0}$ & $\mathbf{2 5 , 8 1 9}$ & $\mathbf{1 0 0 , 0}$ & $\mathbf{1 0 0 , 0}$ & $\mathbf{1 0 0 , 0}$ \\
\hline \multirow{3}{*}{04} & E. erythropappus & 160 & 0,467 & 6,3 & 8,9 & 7,6 \\
& Outras & 2365 & 4,769 & 93,7 & 91,1 & 92,4 \\
& Total & $\mathbf{2 5 2 5}$ & $\mathbf{5 , 2 3 6}$ & $\mathbf{1 0 0 , 0}$ & $\mathbf{1 0 0 , 0}$ & $\mathbf{1 0 0 , 0}$ \\
\hline \multirow{3}{*}{05} & E. erythropappus & 620 & 17,198 & 12,2 & 46,4 & 29,3 \\
& Outras & 4465 & 19,907 & 87,8 & 53,6 & 70,7 \\
& Total & $\mathbf{5 0 8 5}$ & $\mathbf{3 7 , 1 0 5}$ & $\mathbf{1 0 0 , 0}$ & $\mathbf{1 0 0 , 0}$ & $\mathbf{1 0 0 , 0}$ \\
\hline
\end{tabular}

R. Árvore, Viçosa-MG, v.31, n.4, p.667-677, 2007 


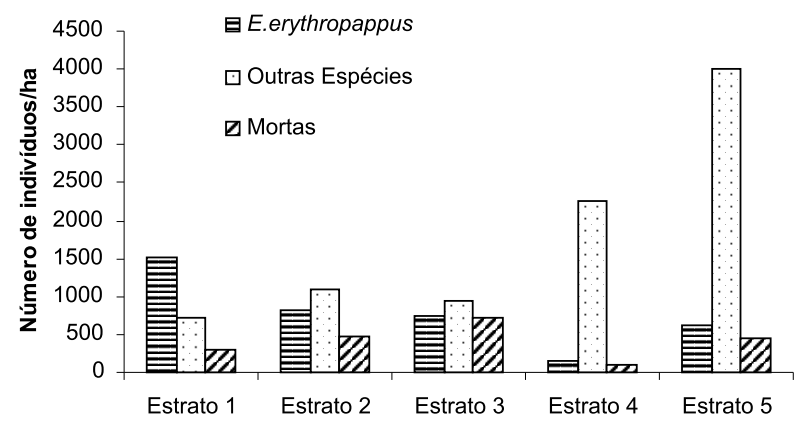

Figura 1 - Distribuição do número de indivíduos por hectare de E. erythropappus, outras espécies e mortas por estrato em setembro de 2000, no Parque Estadual do Itacolomi, em Ouro Preto, MG.

Figure 1 -Distribution of number of individuals per hectare, E. erythropappus, other species and dead trees by stratum in September 2000, Itacolomi State Park, Ouro Preto, $M G$.

A dominância absoluta (DoA), estimada em $m^{2}$ $h a^{-1}$, por área (estrato) foi: Área 1, 20,891 (18,130 $\mathrm{m}^{2}$ $h a^{-1}$ de E. erythropappus e 2,761 $\mathrm{m}^{2} h a^{-1}$ de outras espécies); Área 2, 18,898 (11,745 $\mathrm{m}^{2} h \mathrm{a}^{-1} \mathrm{de}$ E. erythropappus e $7,153 \mathrm{~m}^{2} h a^{-1}$ de outras espécies); Área 3, 25,819 (16,824 $\mathrm{m}^{2} h a^{-1}$ de E. erythropappus e 8,995 $\mathrm{m}^{2} \mathrm{ha}^{-1}$ de outras espécies); Área 4, 5,236 (0,467 $\mathrm{m}^{2} \mathrm{ha}^{-1}$ de E. erythropappus e 4,769 $\mathrm{m}^{2}$ $h a^{-1}$ de outras espécies); e Área 5, 37,105 (17,198 $m^{2} h a^{-1}$ de E. erythropappus e 19,907 $\mathrm{m}^{2} h \mathrm{a}^{-1}$ de outras espécies). Maiores valores de DoA foram observados nas Áreas 1, 5 e 3. A Área 1 sobressaiu em decorrência da maior DA, ao passo que na Área 5 predominam os indivíduos de maiores áreas seccionais de E. erythropappus. Com exceção da Área 1, maiores valores de DoA foram observados em outras espécies (Quadro 1). O solo da Área 1 é bastante raso, com afloramentos rochosos, o que dificulta a regeneração de outras espécies. Em razão disso, nesse tipo de área a candeia predomina e desenvolve bem sua forma específica.

\subsection{Estrutura Vertical}

Os indivíduos arbóreos das comunidades florestais (Áreas 1, 2, 3, 4 e 5) foram, em geral, agrupados em dois estratos de altura total, porém os limites dos estratos verticais diferiram de uma comunidade para outra (Quadro 2). Nas Áreas 1, 2 e 4, o estrato 1 ( $\mathbf{E}_{1}$ ) compreendeu os indivíduos com altura total $(\mathbf{H t})$ menor do que $5 \mathrm{~m}$ e no estrato $2\left(\mathbf{E}_{2}\right)$ foram reunidos os indivíduos com Ht superior ou igual a $5 \mathrm{~m}$, (Quadro 2). Nas Áreas 3 e 5, o estrato $1\left(\mathbf{E}_{1}\right)$ compreendeu os indivíduos com Ht menor do que $7 \mathrm{~m}$ e no estrato $2\left(\mathbf{E}_{2}\right)$ foram reunidos os indivíduos com $\mathbf{H t}$ superior ou igual a 7 m, Na Área 3, a altura total média foi superior ou igual a 5,37 m e na Área 5, superior ou igual a 9,22 m. A proporção do número de árvores por hectare (DA) de E. erythropappus das outras espécies, nos Estratos 1 e 2, foi de aproximadamente 1:3 e $4: 1$, na Área 1; $2: 1$ e $1: 1$, na Área $2 ; 1: 19$ e $1: 1$, na Área 3; $1: 9$ e 1:43, na Área 4; e 1:129 e 1:4, na Área 5. Na Área 1 predominaram indivíduos de candeia no estrato 2 , conferindo-lhe maiores valores de posição sociológica. Nas outras áreas, maiores valores de posição sociológica foram observados nas outras espécies. Em geral, os resultados da estrutura vertical confirmam que as referidas comunidades são constituídas, principalmente, por espécies heliófitas, o que é típico de formações florestais pioneiras.

\subsection{Estrutura Interna}

O número de indivíduos expressos em porcentagem, por hectare, de E. erythropappus encontra-se distribuído, por área e por classe de qualidade de fuste (Quadro $3)$, da seguinte forma: na Área 1, QF1 (17,0\%), QF2 (62,3\%) e QF3 (20,7\%); na Área 2, QF1 (53,6\%), QF2 (42,2\%) e QF3 (4,2\%); na Área 3, QF1 $(26,5 \%)$, QF2 (45,0\%) e QF3 (28,5\%); na Área 4, QF1 $(28,1 \%)$, QF2 (50,0\%) e QF3 (21,9\%); e na Área 5, QF1 (69,4\%), QF2 (16,9\%) e QF3 (13,7\%). Observou-se que é no ambiente de mata natural que os indivíduos de E. erythropappus apresentam fustes de melhor qualidade, isto é, quando assumem a forma florestal.

A compartimentação da área basal por hectare de E. erythropappus, por área e por classe de qualidade de fuste (Quadro 4), foi a seguinte: QF1 (16,8\%), QF2 (64,9\%) e QF3 (18,3\%), na Área 1; QF1 (61,3\%), QF2 $(36,9 \%)$ e QF3 $(1,8 \%)$, na Área 2; QF1 $(20,7 \%)$, QF2 $(39,8 \%)$ e QF3 $(39,5 \%)$, na Área 3; QF1 $(18,0 \%)$, QF2 (65,6\%) e QF3 (16,4\%), na Área 4; e QF1 (61,8\%), QF2 $(15,8 \%)$ e QF3 $(22,4 \%)$, na Área 5.

Na Área 1, a classe de qualidade de fuste 2 (QF2) englobou o maior número total de indivíduos $(50,19 \%)$, e 74,5\% foram de indivíduos de E. erythropappus. $\mathrm{Na}$ Área 1 predominaram indivíduos de E. erythropappus e o sub-bosque é relativamente limpo e com baixa competição por luz; dessa forma, os indivíduos dessa espécie são bastante ramificados, sem o fuste bem definido.

R. Árvore, Viçosa-MG, v.31, n.4, p.667-677, 2007 
Quadro 2 - Distribuição do número de árvores por hectare de E. erythropappus e de outras espécies por estrato de altura total nas áreas 1, 2 e 4. Em que: $\mathrm{E}_{1}=$ Estrato $1(\mathrm{Ht}<5,0 \mathrm{~m})$ e $\mathrm{E}_{2}=$ Estrato $2(\mathrm{Ht} \geq 5,0 \mathrm{~m}) ; \mathrm{PSA}=$ Posição Sociológica Absoluta; e PSR = Posição Sociológica Relativa, em setembro de 2000, no Parque Estadual do Itacolomi, em Ouro Preto, MG

Table 2 - Distribution of tree number per hectare, E. erythropappus and other species, per total height stratum in the sites 1, 2 and 4. Where: $E_{1}=$ Stratum $1(H t<5,0 \mathrm{~m})$ and $E_{2}=$ Stratum $(H t \geq 5,0 \mathrm{~m}) ; \mathrm{PSA}=$ Absolute Sociologic Position, and PSR = Relative Sociologic Position in September 2000, Itacolomi State Park, Ouro Preto-MG

\begin{tabular}{|c|c|c|c|c|c|}
\hline \multirow[t]{2}{*}{ Área } & \multirow[t]{2}{*}{ Espécie } & \multicolumn{2}{|c|}{ Estrato } & \multirow[t]{2}{*}{ PSA } & \multirow[t]{2}{*}{ PSR } \\
\hline & & $\mathrm{E}_{1}$ & $\mathrm{E}_{2}$ & & \\
\hline \multirow{3}{*}{01} & E. erythropappus & 260 & 1265 & 875,266 & 65,6 \\
\hline & Outras Espécies & 725 & 290 & 458,691 & 34,4 \\
\hline & Total & 985 & 1555 & 1333,957 & 100,0 \\
\hline \multirow{3}{*}{02} & E. erythropappus & 60 & 770 & 566,080 & 40,2 \\
\hline & Outras Espécies & 625 & 930 & 842,400 & 59,8 \\
\hline & Total & 685 & 1770 & 1408,480 & 100,0 \\
\hline \multirow{3}{*}{03} & E. erythropappus & 50 & 705 & 439,752 & 35,1 \\
\hline & Outras & 930 & 735 & 813,967 & 64,9 \\
\hline & Total & 980 & 1440 & 1253,719 & 100,0 \\
\hline \multirow{3}{*}{04} & E. erythropappus & 135 & 25 & 86,861 & 6,8 \\
\hline & Outras Espécies & 1285 & 1080 & 1195,287 & 93,2 \\
\hline & Total & 1420 & 1105 & 1282,149 & 100,0 \\
\hline \multirow{3}{*}{05} & E. erythropappus & 15 & 605 & 379,326 & 14,1 \\
\hline & Outras & 1930 & 2535 & 2303,589 & 85,9 \\
\hline & Total & 1945 & 3140 & 2682,915 & 100,0 \\
\hline
\end{tabular}

Quadro 3 - Número de árvores por hectare de E. erythropappus e demais espécies, por classe de qualidade de fuste, em que QF1 = Qualidade de Fuste 1 (80 - 100\% de aproveitamento do fuste comercial); QF2 = Qualidade de Fuste $2(50-79 \%$ de aproveitamento do fuste comercial); QF3 = Qualidade de Fuste 3 (0 - 49\% de aproveitamento do fuste comercial); QFA = Qualidade de Fuste Absoluta; e QFR = Qualidade de Fuste Relativa, amostradas nas áreas 1, 2, 3, 4 e 5, em setembro de 2000, no Parque Estadual do Itacolomi, Ouro Preto, MG

Table 3 - Number of tree per hectare, E. erythropappus and other species, per trunk quality class, where QF $1=T r u n k$ Quality 1 (80-100\% profitable commercial trunk), QF2 = Trunk Quality 2(50 - 79\% profitable commercial trunk), QF3 = Trunk Quality $3(0-49 \%$ profitable commercial trunk), $Q F A=A$ bsolute Trunk Quality and QFR $=$ Relative Trunk Quality, sampled in the sites 1,2,3,4 and 5, in September 2000, Itacolomi State Park, Ouro Preto-MG

\begin{tabular}{llccccc}
\hline Área & Espécie & QF1 & QF2 & QF3 & QFA & QFR \\
\hline \multirow{3}{*}{01} & E. erythropappus & 260 & 950 & 315 & 621,841 & 64,7 \\
& Outras & 290 & 325 & 400 & 338,533 & 35,3 \\
& Total & $\mathbf{5 5 0}$ & $\mathbf{1 2 7 5}$ & $\mathbf{7 1 5}$ & $\mathbf{9 6 0 , 3 7 4}$ & $\mathbf{1 0 0 , 0}$ \\
\hline \multirow{3}{*}{02} & E. erythropappus & 445 & 350 & 35 & 352,264 & 37,0 \\
& Outras & 590 & 705 & 260 & 600,053 & 63,0 \\
& Total & $\mathbf{1 0 3 5}$ & $\mathbf{1 0 5 5}$ & $\mathbf{2 9 5}$ & $\mathbf{9 5 2 , 3 1 7}$ & $\mathbf{1 0 0 , 0}$ \\
\hline \multirow{3}{*}{03} & E. erythropappus & 200 & 340 & 215 & 251,002 & 30,8 \\
& Outras & 700 & 465 & 500 & 562,738 & 69,2 \\
& Total & $\mathbf{9 0 0}$ & $\mathbf{8 0 5}$ & $\mathbf{7 1 5}$ & $\mathbf{8 1 3 , 7 4 0}$ & $\mathbf{1 0 0 , 0}$ \\
\hline \multirow{3}{*}{04} & E. erythropappus & 45 & 80 & 35 & 57,881 & 5,9 \\
& Outras & 1075 & 955 & 335 & 917,376 & 94,1 \\
& Total & $\mathbf{1 1 2 0}$ & $\mathbf{1 0 3 5}$ & $\mathbf{3 7 0}$ & $\mathbf{9 7 5 , 2 5 7}$ & $\mathbf{1 0 0 , 0}$ \\
\hline \multirow{3}{*}{05} & E. erythropappus & 430 & 105 & 85 & 318,702 & 12,4 \\
& Outras & 2945 & 1095 & 425 & 2255,679 & 87,6 \\
& Total & $\mathbf{3 3 7 5}$ & $\mathbf{1 2 0 0}$ & $\mathbf{5 1 0}$ & $\mathbf{2 5 7 4 , 3 8 1}$ & $\mathbf{1 0 0 , 0}$ \\
\hline
\end{tabular}

R. Árvore, Viçosa-MG, v.31, n.4, p.667-677, 2007 
Quadro 4 - Área basal $\left(m^{2} h a^{-1}\right)$ para E. erythropappus e demais espécies por classe de qualidade de fuste, em que QF1 = Qualidade de Fuste 1 ( $80-100 \%$ de aproveitamento do fuste comercial); QF2 = Qualidade de Fuste 2 (50 $-79 \%$ de aproveitamento do fuste comercial); QF3 = Qualidade de Fuste 3 (0 - 49\% de aproveitamento do fuste comercial); e QFT = Qualidade de Fuste Total, amostradas nas áreas 1, 2, 3, 4 e 5, em setembro de 2000, no Parque Estadual do Itacolomi, em Ouro Preto, MG

Table 4-Basal area $\left(\mathrm{m}^{2} h \mathrm{a}^{-1}\right)$, for E. erythropappus and other species, and per trunk quality class, where QF $1=T r u n k$ Quality 1 ( $80-100 \%$ profitable commercial trunk), QF2 = Trunk Quality 2 (50-79\% profitable commercial trunk), QF3 = Trunk Quality $3(0-49 \%$ profitable commercial trunk), $Q F A=A$ ssolute Trunk Quality and $Q F R$ $=$ Relative Trunk Quality, sampled in the sites 1,2,3,4 and 5, in September 2000, Itacolomi State Park, Ouro Preto$M G$

\begin{tabular}{|c|c|c|c|c|c|}
\hline \multirow[t]{2}{*}{ Área } & \multirow[t]{2}{*}{ Espécie } & \multicolumn{3}{|c|}{ Qualidade de fuste (QF) } & \multirow[t]{2}{*}{ QFT } \\
\hline & & QF1 & QF2 & QF3 & \\
\hline \multirow{3}{*}{01} & E. erythropappus & 3,0409 & 11,7607 & 3,3285 & 18,1301 \\
\hline & Outras & 0,5574 & 0,8738 & 1,3299 & 2,7611 \\
\hline & Total & 3,5983 & 12,6345 & 4,6584 & 20,8912 \\
\hline \multirow{3}{*}{02} & E. erythropappus & 7,2009 & 4,3353 & 0,2089 & 11,7451 \\
\hline & Outras & 2,0108 & 2,3849 & 2,7571 & 7,1528 \\
\hline & Total & 9,2117 & 6,7202 & 2,9660 & 18,8979 \\
\hline \multirow{3}{*}{03} & E. erythropappus & 3,4873 & 6,6997 & 6,6373 & 16,8243 \\
\hline & Outras & 3,3232 & 1,7360 & 3,9356 & 8,9949 \\
\hline & Total & 6,8105 & 8,4357 & 10,5729 & 25,8192 \\
\hline \multirow{3}{*}{04} & E. erythropappus & 0,0838 & 0,3064 & 0,0765 & 0,4668 \\
\hline & Outras & 2,0545 & 2,0317 & 0,6835 & 4,7696 \\
\hline & Total & 2,1383 & 2,3381 & $\mathbf{0 , 7 6 0 0}$ & 5,2364 \\
\hline \multirow{3}{*}{05} & E. erythropappus & 10,6310 & 2,7234 & 3,8433 & 17,1977 \\
\hline & Outras & 12,9097 & 4,6412 & 2,3567 & 19,9076 \\
\hline & Total & 23,5407 & 7,3646 & 6,2000 & 37,1053 \\
\hline
\end{tabular}

No Quadro 5, apresenta-se a distribuição do volume absoluto (VoA), estimado em $\mathrm{m}^{3} / \mathrm{ha}$, por classe de qualidade de fuste. De acordo com Pedralli (1997), os indivíduos de E. erythropappus apresentam porte maior na sua forma florestal, observando-se que na Área 5 estava contido o maior volume absoluto da espécie $\left(71,4805 \mathrm{~m}^{3} / \mathrm{ha}\right)$. Na Área 4, que também estava na forma florestal, isso não ocorreu devido à baixa densidade e dominância apresentada pela espécie.

A distribuição de volume de fuste de E. erythropappus por área e por classe de qualidade de fuste (Quadro 5) encontrava-se assim distribuída: na Área 1, 61,3\% do volume total por hectare estava na classe de QF2 e E. erythropappus representava $88,0 \%$ do volume total por hectare, do qual 17,0, 65,3 e $17,7 \%$, pertenciam às classes de QF1, QF2 e QF3, respectivamente; na Área 2, predominavam os volumes na classe de QF1 (50,9\%), e E. erythropappus totalizava
$63,5 \%$ do volume total por hectare, $62,9 \%$ na classe QF1, 35,6\% na QF2 e 1,5\% na QF3; na Área 3, E. erythropappus contribuiu com $65,5 \%$ do volume total por hectare, tendo, respectivamente, $20,6 \%, 39,3 \%$ e 40,1\% nas classes QF1, QF2 e QF3; na Área 4, os indivíduos de Baccharis sp. perfizeram $62,3 \%$ do volume total por hectare, enquanto E. erythropappus somou apenas 7,0\%; e na Área 5, E. erythropappus contribuiu com $47,0 \%$ do volume por hectare, sendo $61,5 \%$ na classe QF1, 16,0\% na QF2 e 22,5\% na QF3.

\subsection{Estrutura Diamétrica}

Observaram-se maiores valores de DA na classe de DAP de 3-6 cm. A distribuição diamétrica apresentava tendência geral de "J-invertido", o que é uma característica da estrutura inequiiânea (Figura 2). A espécie E. erythropappus distribuía-se em todas as classes de DAP, com maiores valores de DA concentrados nas

R. Árvore, Viçosa-MG, v.31, n.4, p.667-677, 2007 
classes de DAP de 3-6 cm e 6-8 cm (Área 1 e Área 4), e nas áreas 2 e 3 os maiores valores de DA estavam na classe de DAP de 10-13 cm (Quadro 6). Conforme Carvalho et al. (1995), a E. erythropappus apresentava maior densidade de indivíduos nas menores classes de DAP, com decréscimo gradual em direção às classes maiores, o que indica boa reprodução e recrutamento contínuo. Na Área 5, observaram-se maiores valores de DA de E. erythropappus na classe de DAP de $20-30 \mathrm{~cm}$, sendo a única área com indivíduos na classe de DAP de 50$60 \mathrm{~cm}$ e ausência na classe de DAP de $3-6 \mathrm{~cm}$, fato que se deve ao estádio sucessional que estava mais avançado nessa área do que nas demais, o que ocasiona maior competição pelo fator luz de E. erythropappus com outras espécies. Na Área 4, foram encontrados indivíduos apenas em quatro classes de DAP, pertencendo à maioria à classe de DAP de 3-6 cm, o que indica que essa área estava em estádio inicial de sucessão secundária.

Quanto às estimativas do número de moirões de candeia por classe de DAP, observa-se, no (Quadro 7), que na Área 3 (candeial) se encontrava o maior número $(37,8 \%)$ de moirões por hectare, estando a maioria desses $(21,8 \%)$ na classe de DAP de $10-13$ cm. A Área $\mathbf{1}$ (candeial) apresentou 10,8\% do número total de moirões concentrados na classe de DAP 6-8 cm. Na Área 2 (candeial) ocorreu 19,3\% do total de moirões, sendo $26,7 \%$ presentes na classe de DAP $10-13 \mathrm{~cm}$. Na Área 4 (ocorrência rara de candeia) houve a presença de moirões apenas na primeira classe de DAP (3-6 cm). A Área 5 contribuiu com $31,4 \%$ do total de moirões de todas as áreas, e 32,8\% desses pertenciam à classe de 20-30 cm de DAP. A análise conjunta das cinco áreas apontou que o maior porcentual $(20,8 \%)$ de moirões se encontrava na classe de $10-13 \mathrm{~cm}$ de DAP, cujos resultados batiam com aqueles obtidos por Perez et al. (2004).

Os resultados do teste Qui-quadrado indicaram que a distribuição da densidade $\left(n h a^{-1}\right)$ por classe de DAP das áreas de estudo diferiram significativamente $(\mathrm{P}<0,05)$, considerando-se todas as espécies. O mesmo ocorreu nas distribuições da densidade $\left(n h a^{-1}\right)$ por classe de DAP da espécie E. erythropappus das áreas de estudo, em todas as combinações de comparação entre as áreas.

Quadro 5 - Volume $\left(m^{3} h a^{-1}\right)$ de E. erythropappus e demais espécies e por classe de qualidade de fuste, em que QF1 = Qualidade de Fuste 1 (80-100\% de aproveitamento do fuste comercial); QF2 = Qualidade de Fuste 2 (50-79\% de aproveitamento do fuste comercial); QF3 = Qualidade de Fuste 3 (0-49\% de aproveitamento do fuste comercial); QFT = Qualidade de Fuste Total, amostradas nas áreas 1, 2, 3, 4 e 5, em setembro de 2000, no Parque Estadual do Itacolomi, em Ouro Preto, MG

Table 5 - Volume $\left(\mathrm{m}^{3} \mathrm{ha}^{-1}\right)$, for E. erythropappus and other species, and per trunk quality class, where QF $1=$ Trunk Quality 1 ( $80-100 \%$ profitable commercial trunk), QF2 = Trunk Quality 2(50 - 79\% profitable commercial trunk), QF3 = Trunk Quality $3(0-49 \%$ profitable commercial trunk), QFA = Absolute Trunk Quality and $Q F R=$ Relative Trunk Quality, sampled in the sites 1,2,3,4 and 5, in September 2000, Itacolomi State Park, Ouro Preto-MG

\begin{tabular}{|c|c|c|c|c|c|}
\hline Área & Espécie & QF1 & QF2 & QF3 & QFT \\
\hline & E. erythropappus & 12,1472 & 46,6698 & 12,6635 & 71,4805 \\
\hline \multirow[t]{2}{*}{01} & Outras & 2,2124 & 3,1527 & 4,3797 & 9,7449 \\
\hline & Total & 14,3596 & 49,8226 & 17,0432 & 81,2254 \\
\hline \multirow{3}{*}{02} & E. erythropappus & 32,2837 & 18,3034 & 0,7792 & $\overline{51,3663}$ \\
\hline & Outras & 8,9079 & 10,3936 & 10,2495 & 29,5509 \\
\hline & Total & 41,1916 & 28,6970 & $\mathbf{1 1 , 0 2 8 7}$ & 80,9172 \\
\hline \multirow{3}{*}{03} & E. erythropappus & 17,9071 & 34,2082 & 34,9586 & 87,0738 \\
\hline & Outras & 18,7575 & 8,8978 & 18,0958 & 45,7511 \\
\hline & Total & 36,6646 & 43,1059 & 53,0544 & 132,8250 \\
\hline \multirow{3}{*}{04} & E. erythropappus & 0,2296 & 0,9421 & 0,1910 & 1,3627 \\
\hline & Outras & 8,1112 & 7,5586 & 2,3608 & 18,0306 \\
\hline & Total & 8,3409 & 8,5006 & 2,5518 & 19,3933 \\
\hline \multirow{3}{*}{05} & E. erythropappus & 60,1363 & 15,6021 & 22,0130 & 97,7514 \\
\hline & Outras & 73,6999 & 25,6134 & 10,8115 & 110,1248 \\
\hline & Total & 133,8362 & 41,2155 & 32,8245 & 207,8762 \\
\hline
\end{tabular}

R. Árvore, Viçosa-MG, v.31, n.4, p.667-677, 2007 

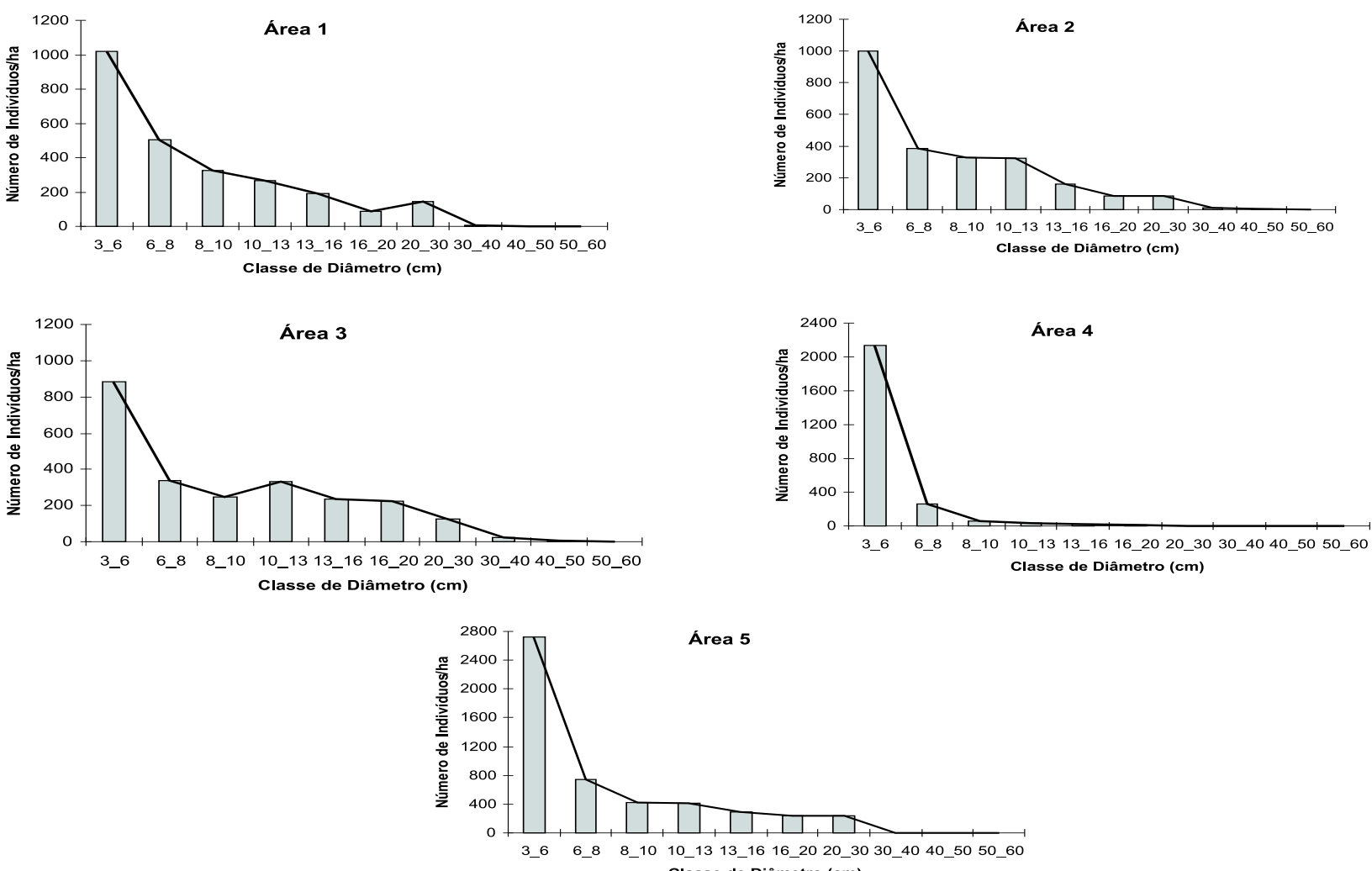

Figura 2 - Distribuição do número total de indivíduos por hectare, por classe de diâmetro, nas cinco áreas estudadas, em setembro de 2000, no Parque Estadual do Itacolomi, em Ouro Preto, MG.

Figure 2 -Distribution of total number of individuals per hectare and diameter class, for the 5 studied sites in September 2000, Itacolomi State Park, Ouro Preto-MG.

Quadro 6 - Número de árvores (DA), área basal (DoA) e volume total com casca por hectare (VoA) e por classe de DAP (cm) de E. erythropappus nas áreas 1, 2, 3, 4 e 5, em setembro de 2000, no Parque Estadual do Itacolomi, em Ouro Preto, MG

Table 6 - Number of trees (DA), basal area (DoA), and total volume with bark (VoA) per hectare and per DAP(DBH) class (cm) for E. erythropappus in the sites 1,2,3,4 and 5, in September 2000, Itacolomi State Park, Ouro Preto-MG

\begin{tabular}{|c|c|c|c|c|c|c|c|c|c|c|c|c|}
\hline \multirow[t]{2}{*}{ Área } & \multirow[t]{2}{*}{ Variável } & \multicolumn{10}{|c|}{ Classe de DAP $(\mathrm{cm})$} & \multirow[t]{2}{*}{ Total } \\
\hline & & $3 \_6$ & $6 \_8$ & $8 \_10$ & 10_13 & $13 \_16$ & 16_20 & 20_30 & 30_40 & $40 \_50$ & $50 \_60$ & \\
\hline \multirow{3}{*}{01} & DA & 310 & 310 & 270 & 235 & 165 & 85 & 145 & 5 & & & 1525 \\
\hline & DoA & 0,572 & 1,220 & 1,717 & 2,422 & 2,675 & 2,168 & 6,979 & 0,378 & & & 18,130 \\
\hline & VoA & 1,849 & 4,463 & 6,429 & 9,106 & 10,721 & 8,412 & 29,141 & 1,358 & & & 71,481 \\
\hline \multirow{3}{*}{02} & DA & 80 & 115 & 140 & 210 & 125 & 80 & 75 & 5 & & & 830 \\
\hline & DoA & 0,157 & 0,430 & 0,890 & 2,181 & 2,060 & 1,971 & 3,557 & 0,499 & & & 11,745 \\
\hline & VoA & 0,547 & 1,509 & 3,543 & 8,972 & 8,810 & 9,165 & 16,598 & 2,222 & & & 51,366 \\
\hline \multirow{3}{*}{03} & DA & 35 & 35 & 65 & 165 & 160 & 145 & 120 & 25 & 5 & & 755 \\
\hline & DoA & 0,074 & 0,128 & 0,425 & 1,736 & 2,654 & 3,735 & 5,076 & 2,318 & 0,676 & & 16,824 \\
\hline & VoA & 0,266 & 0,505 & 2,038 & 8,610 & 13,524 & 19,490 & 26,665 & 12,309 & 3,667 & & 87,074 \\
\hline \multirow{3}{*}{04} & DA & 130 & 15 & & 10 & & 5 & & & & & 160 \\
\hline & DoA & 0,183 & 0,061 & & 0,111 & & 0,112 & & & & & 0,467 \\
\hline & VoA & 0,451 & 0,174 & & 0,329 & & 0,408 & & & & & 1,363 \\
\hline \multirow{3}{*}{05} & DA & & 10 & 60 & 110 & 100 & 130 & 200 & & 5 & 5 & 620 \\
\hline & DoA & & 0,039 & 0,387 & 1,133 & 1,647 & 3,175 & 8,865 & & 0,802 & 1,150 & 17,198 \\
\hline & VoA & & 0,166 & 1,855 & 5,793 & 8,636 & 17,755 & 51,703 & & 5,290 & 6,554 & 97,751 \\
\hline
\end{tabular}


Quadro 7 - Estimativa do número de moirões de E. erythropappus por hectare e por classe de DAP das cinco áreas amostradas em setembro de 2000, no Parque Estadual do Itacolomi, em Ouro Preto, MG

Table 7 - Estimate of number offence posts of E. erythropappus per hectare and DAP $(D B H)$ class (cm) for the five sampled sites, in September 2000, Itacolomi State Park, Ouro Preto-MG

\begin{tabular}{|c|c|c|c|c|c|c|c|c|c|c|c|}
\hline \multirow[t]{2}{*}{ Área } & \multicolumn{10}{|c|}{ Classe de DAP $(\mathrm{cm})$} & \multirow[t]{2}{*}{ Total } \\
\hline & $3 \_6$ & $6 \_8$ & $8 \_10$ & $10 \_13$ & $13 \_16$ & 16_20 & $20 \_30$ & $30 \_40$ & $40 \_50$ & $50 \_60$ & \\
\hline 1 & 25 & 65 & 60 & 35 & 10 & 5 & 10 & & & & 210 \\
\hline 2 & 20 & 55 & 85 & 100 & 60 & 30 & 25 & & & & 375 \\
\hline 3 & 35 & 35 & 65 & 160 & 155 & 145 & 110 & 25 & 5 & & 735 \\
\hline 4 & 15 & & & & & & & & & & 15 \\
\hline 5 & & 5 & 55 & 110 & 100 & 130 & 200 & & 5 & 5 & 610 \\
\hline Total & 95 & 160 & 265 & 405 & 325 & 310 & 345 & 25 & 10 & 5 & 1945 \\
\hline
\end{tabular}

\section{CONCLUSÕES}

A Área 1 apresentou o maior número de indivíduos de E. erythropappus por hectare e a maior dominância absoluta $\left(m^{2} h a^{-1}\right)$, o volume absoluto $\left(m^{3} h a^{-1}\right)$ foi maior na Área 5, por conter indivíduos de maior porte.

Em todas as áreas analisadas foram gerados dois estratos de altura total para os indivíduos analisados, e os limites dos estratos verticais diferiram de uma área para outra.

A Área 5 apresentou maior quantidade de volume com fuste bom para a espécie E. erythropappus, enquanto na Área 4 a contribuição de volume por indivíduos dessa espécie foi a mais baixa entre ás áreas analisadas.

Em todas áreas analisadas, os indivíduos de E. erythropappus estão concentrados nas menores classes de diâmetro.

Analisando as cinco áreas em conjunto, constatouse que a maior quantidade de moirões estava na classe de DAP de 10 a $13 \mathrm{~cm}$, num total 405 moirões.

\section{REFERÊNCIAS}

\section{ASSOCIAÇÃO BRASILEIRA DE NORMAS} TÉCNICAS - ABNT. Mourões de madeira preservada para cercas. NBR 9480 , Agosto de 1986.

AMARAL, P. et al. Floresta para sempre: um manual para a produção de madeira na Amazônia. Belém: IMAZON, 1998. 137p.

ANTUNES, F. Z. Caracterização climática do Estado de Minas Gerais. Informe Agropecuário, v.12, n.138, p.9-13, 1986.

R. Árvore, Viçosa-MG, v.31, n.4, p.667-677, 2007
ARAÚJO, L. C. Vanillosmopsis erythropappa (DC.) Sch. Bip. e sua exploração florestal. 1994. 60f. Dissertação (Professor Catedrático) - Escola Nacional de Agricultura, Rio de Janeiro, 1994.

CARVALHO, D. A. et al. Estrutura diamétrica e vertical de uma floresta ripária no Alto Rio Grande (Bom Sucesso) - Estado de Minas Gerais.

Revista Árvore, v.19, n.4, p.572-586, 1995.

EMPRESA BRASILEIRA DE PESQUISA AGROPECUÁRIA - EMPRAPA. Sistema Brasileiro de Classificação de Solos. Rio de Janeiro: 1999. 412p.

FUNDAÇÃO CENTRO TECNOLÓGICO DE MINAS GERAIS - CETEC.

Desenvolvimento de equações volumétricas aplicáveis ao manejo sustentado de florestas nativas do estado de Minas Gerais e outras regiões do país. Belo Horizonte, Fundação Centro Tecnológico de Minas Gerais, 1995.

FUNDAÇÃO CENTRO TECNOLÓGICO DE MINAS GERAIS - CETEC. Ecofisiologia da

Candeia. Belo Horizonte: SAT/CETEC, 1996. 103p. (Relatório Técnico).

FUNDAÇÃO ESTADUAL DO MEIO AMBIENTE FEAM. Plano de manejo da estação ecológica do Tripuí, Ouro Preto, MG. Belo Horizonte: 1995. v.1. 69p.

INSTITUTO ESTADUAL DE FLORESTAS - IEF. Parques Estaduais. Disponível em: < http:/ /www.ief.mg.gov.br>. Acesso em: 19 de dezembro 2004. 
LAMPRECHT, H. Ensayo sobre la estructura floristica de la parte suroriental del bosque universitario "el caimital", Estado Barinas. Revista Forestal Venezolana, v.7, n.10/11, 77-119, 1964.

PEDRALLI, G. Estrutura diamétrica, vertical e análise do crescimento da "candeia" (Vanillosmopsis erythropappa $\mathrm{Sch}$. Bip.) na Estação Ecológica do Tripuí, Ouro Preto - MG. Revista Árvore, v.21, n.2, p.301-306, 1997.
PÉREZ, J. F. M. et al. Sistema de manejo para candeia (Eremanthus erythropappa (DC.) MacLeish). Revista Cerne, v.10, n.2, p.257-273, 2004.

SOUZA, D. R. et al. Emprego de análise multivariada para estratificação vertical de florestas inequiâneas. Revista Árvore, v.27, n.1, p.59-63, 2003. 
